\title{
Study of inhomogeneity in thickness of LR 115 detector with SEM and Form Talysurf
}

\author{
C.W.Y. Yip, J.P.Y. Ho, D. Nikezic ${ }^{1}$, K.N. Yu* \\ Department of Physics and Materials Science, City University of Hong Kong, Tat Chee Avenue Kowloon Tong, Kowloon, Hong Kong
}

Received 21 October 2002; accepted 24 April 2003

\begin{abstract}
Long-term measurements of radon progeny concentrations using Solid-State nuclear tract detector are being actively explored. These measurements depend critically on the thickness of the removed layer during etching. Scanning electron microscope (SEM) observations have identified irregularities in etched LR 115 detectors, such as detachment of the active layer from the substrate and formation of air gaps in the substrate. After discarding these irregularities, by using "Form Talysurf" surface profile measurements, the thickness of the active layers for the LR 115 detector are found to be $11.8 \pm 0.2$ and $5.0 \pm 0.4 \mu \mathrm{m}$ before and after $2 \mathrm{~h}$ of etching, respectively. The coefficient of variation has thus risen from $1.7 \%$ to $8.0 \%$ on etching. The increased inhomogeneity is explained by the formation of track-like damages, which have been observed using Form Talysurf, SEM, optical microscope and atomic force microscope. With this relative large coefficient of variation, the thickness of the active layer in the LR 115 detector cannot be assumed to be homogeneous in general, and the associated uncertainties should be considered carefully when the detector is used for alpha spectroscopy.
\end{abstract}

(c) 2003 Elsevier Ltd. All rights reserved.

Keywords: LR 115; Thickness; Bulk etch

\section{Introduction}

It is established that tracheobronchial deposition of ${ }^{222} \mathrm{Rn}$ progeny in the human body can lead to lung cancer, and people have become increasingly interested in the long-term measurements of ${ }^{222} \mathrm{Rn}$ progeny concentrations. Alpha spectroscopy by solid-state nuclear track detectors (SSNTDs) has been the most popular method proposed for such long-term measurements, and is still being actively studied and explored. The LR 115 detector is one of the most commonly used SSNTDs. All these measurements depend critically on the thickness of the removed layer during etching. For this reason, it is pertinent to identify a direct and an accurate method to determine the thickness of the removed layer of the LR 115 detector, and to study the inhomogeneity in this thickness.

\footnotetext{
* Corresponding author. Tel.: +852-2788-7812; fax: +8522788-7830.

E-mail address: peter.yu@cityu.edu.hk (K.N. Yu).

${ }^{1}$ On leave from University of Kragujevac, Yugoslavia.
}

In most current track studies using SSNTDs, the thickness of the active layers or the detectors themselves are assumed to be homogeneous, which has not been verified rigorously and may not be realistic. The knowledge from the present study can give us an idea of how well such an assumption holds, and provides a base for determining the expected uncertainties in alpha spectroscopy using the LR 115 detector.

\section{Methodology}

Various methods have been proposed or employed for the determination of the amount of bulk etch in SSNTDs. For example, one relied on the difference between detector mass before and after etching, and another was based on measurements of track opening diameters (Durrani and Bull, 1987). Nevertheless, these were indirect measurements. Recently, Nikezić and Janicijević (2002) proposed a method to directly measure the bulk etch for the LR 115 detector based on surface profile measurements. This method is convenient and direct, and represents a good candidate as a 
standardized method for the determination of the thickness of the removed layer of the LR 115 detector, so this method is adopted in the present study.

The LR 115 detectors were purchased from DOSIRAD, France (LR 115 film, Type 2, non-strippable, $12 \mu \mathrm{m}$ red cellulose nitrate on a $100 \mu \mathrm{m}$ clear polyester base). In the present experiments, the detectors were cut with a size of $2 \times 2 \mathrm{~cm}^{2}$. Before etching, a portion of the red cellulose nitrate layer was first removed by a razor to expose the colorless polyester base. The thickness of the active layer was then revealed by the profile of this cliff of active layer over the substrate (Nikezić and Janicijević, 2002).

The detectors before and after etching were measured using an instrument called "Form Talysurf" (Taylor Hobson, Leicester, England), which is based on a laser interferometric transducer. A computer-controlled stylus passes slowly across a surface of specimen during measurements. The data are processed at the same time by the computer to generate an output graph showing the profile of the scanned surface. The accuracy of the instrument is $0.004 \mu \mathrm{m}$.

As a quality assurance procedure, a scanning electron microscope (SEM) (model JSM-820, JEOL) was employed to check whether the etched LR 115 detectors are suitable for Form Talysurf measurements. Some irregularities in the etched LR 115 detectors have been identified, which will be discussed in the next section. These samples will have large inhomogeneities of the active layer, so they will be discarded. The SEM was also used to study the structure of the LR 115 detector before etching, and to compare the texture of the active-layer surface before and after etching.

The homogeneities of the active layer of the LR 115 detector before etching and after $2 \mathrm{~h}$ of etching were investigated. Etching of the detectors was carried out in a $2.5 \mathrm{~N}$ $\mathrm{NaOH}$ solution at $60^{\circ} \mathrm{C}$ using a water bath controlled with a thermostat. The temperature was kept constant with an accuracy of $\pm 1^{\circ} \mathrm{C}$. After $2 \mathrm{~h}$ of etching, the detectors were removed from the etchant and immediately rinsed with distilled water. The mean values and the standard deviations for the thickness of the active layers were obtained through measurements from the Form Talysurf for 10 different positions of the cliff.

\section{Results and discussion}

Fig. 1 is an SEM image of a piece of LR 115 detector before etching, with a portion of the cellulose nitrate layer removed by a razor to expose the polyester base. The thickness of the active layer is given by the height of the cliff of the active layer over the substrate. The trough in front of the cliff is a result of the razor cut into the polyester base of the detector during the removal of the active layer. Nevertheless, this cut will not affect the measurements of the active layer thickness. From Fig. 1, we also have three observations, namely (1) the active-layer surface is smooth, (2) the active layer is tightly glued to the substrate and (3) there is

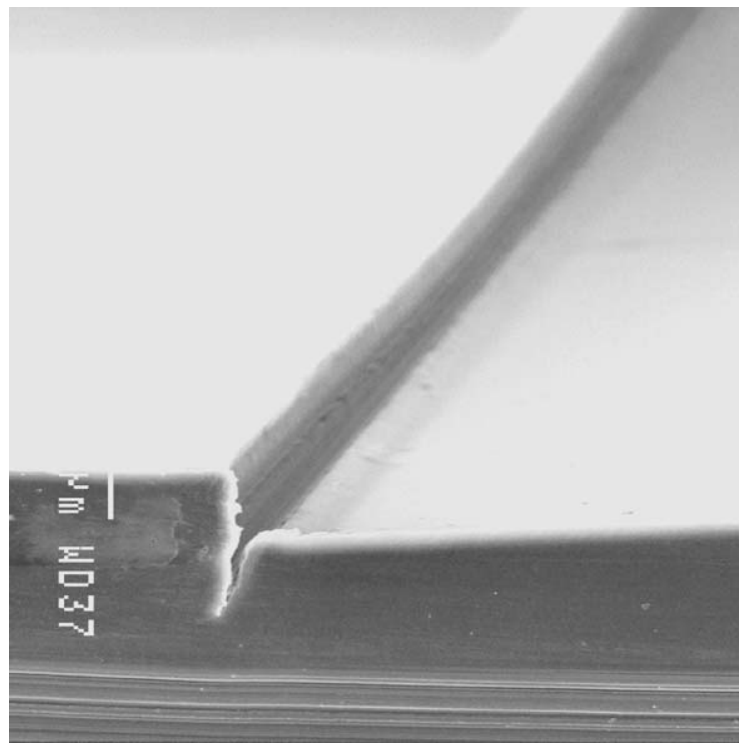

Fig. 1. The cross-sectional view of a piece of LR 115 detector imaged using a scanning electron microscope before etching, showing the cliff of the active layer (on the left) over the substrate (on the right).

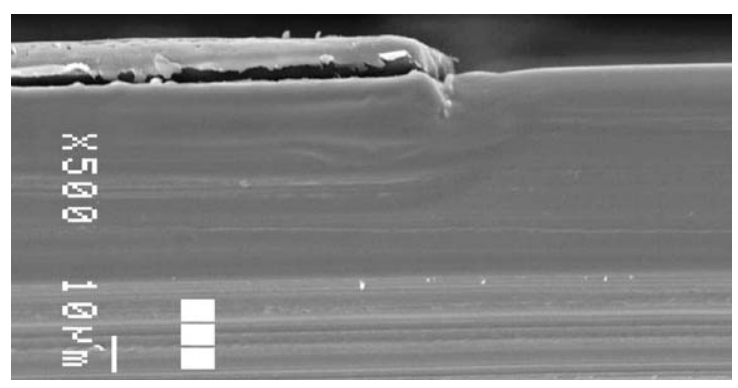

Fig. 2. The cross-sectional view of a piece of LR 115 detector imaged using a scanning electron microscope after $30 \mathrm{~min}$ of etching. The active layer (shown on the left) is partially detached from the substrate.

no air gap in the substrate. These are characteristics of all LR 115 detectors before etching, which, however, might not be of those after etching.

For example, Fig. 2 shows the cross-sectional view of a piece of LR 115 detector imaged using the SEM after $30 \mathrm{~min}$ of etching. In this figure, we can see that the active layer is partially detached from the substrate. Another example is found in Fig. 3 which shows the cross-sectional view of a piece of LR 115 detector imaged using the SEM after $1 \mathrm{~h}$ of etching. In this figure, we notice that large air gaps have been formed in the substrate layer of the detector. These kinds of samples will cause erroneous results in the active-layer measurements, and should be discarded during the quality assurance procedure. 


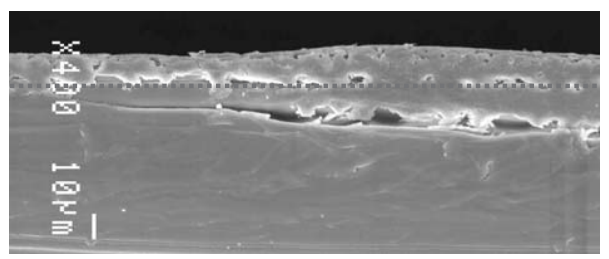

Fig. 3. The cross-sectional view of a piece of LR 115 detector imaged using a scanning electron microscope after $1 \mathrm{~h}$ of etching. Air gaps have been formed in the substrate layer of the detector. The dotted line represents the interface between the active layer and the substrate.

\section{Table 1}

The active-layer thickness for the LR 115 detector before etching and after $2 \mathrm{~h}$ of etching measured by Form Talysurf $(\mu \mathrm{m})$

\begin{tabular}{ll}
\hline & Mean thickness of the active layer $(\mu \mathrm{m})$ \\
\hline Before etching & $11.76 \pm 0.20$ \\
After etching & $4.99 \pm 0.40$ \\
\hline
\end{tabular}

When the samples do not have these irregularities, they can be used to determine the inhomogeneity in thickness of LR 115 detectors. As mentioned before, the mean values and the standard deviations for the thickness of the active layers were obtained for 10 different positions of the cliff. The data are shown in Table 1. The thicknesses of the active layers for the LR 115 detectors are $11.76 \pm 0.20$ and $4.99 \pm 0.40 \mu \mathrm{m}$ before etching and after $2 \mathrm{~h}$ of etching, respectively. Before etching, the standard deviation is $0.2 \mu \mathrm{m}$, which represents the uncertainties caused by the variation of the active layer thickness as well as by the measurement of Form Talysurf. This is the minimum uncertainty of the present method for determining the active-layer thickness. The coefficient of variation, defined by the ratio of the standard deviation to the mean, is $1.7 \%$ for unetched LR 115 detectors. After etching, the standard deviation has increased to $0.4 \mu \mathrm{m}$ and the coefficient of variation has increased to $8.0 \%$.

In the following, the reason behind the increased inhomogeneity in the thickness of LR 115 detector after etching will be explored. Fig. 4 shows the profile of a piece of LR 115 detector after etching given by the Form Talysurf. Here we can see that the surface of the active layer is very rough. A few big troughs are seen with openings of $\sim 4 \mu \mathrm{m}$ and the deepest one has a depth of $\sim 0.37 \mu \mathrm{m}$. In contrast, the surface of the active layer of an unetched LR 115 detector before etching is very smooth in the profile given by the Form Talysurf.

The SEM was also used to compare the LR 115 detector surface before and after etching. A piece of LR 115 detector was masked partially with rubber cement (product no.140, Union Rubber Inc., Trenton) and etched. Fig. 5 shows both the surface of the masked part as well as

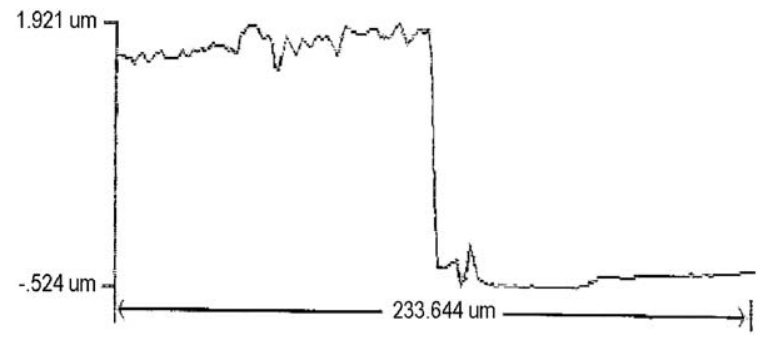

Fig. 4. A profile of a piece of LR 115 detector after etching given by the Form Talysurf, showing the cliff of the active layer (on the left) over the substrate (on the right).

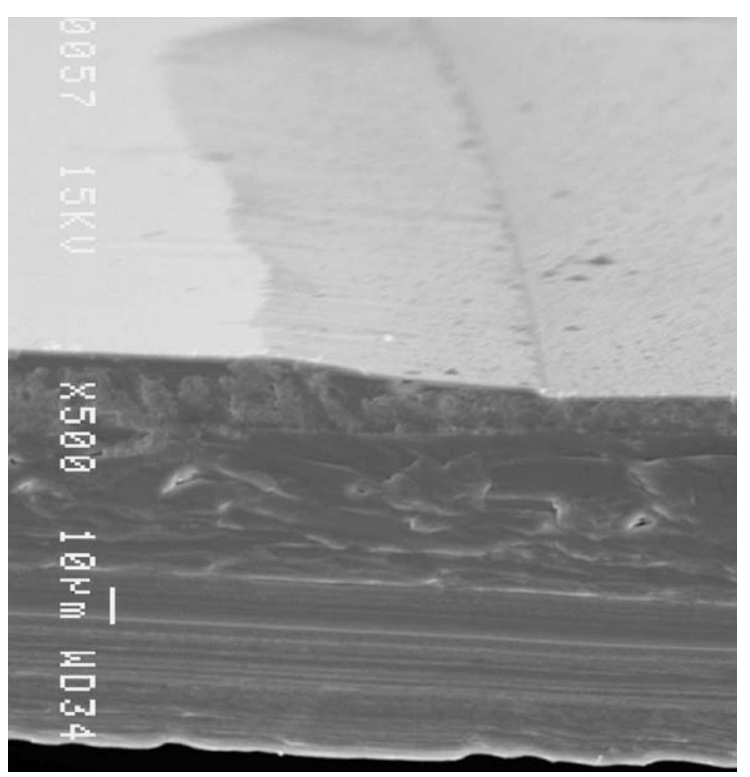

Fig. 5. The cross-sectional view of a piece of LR 115 detector imaged using a scanning electron microscope after etching for $30 \mathrm{~min}$. During etching, the detector was partially masked (on the left).

the exposed part of the detector. The masked part (representing the unetched detector) shows a very smooth surface, while the exposed part (representing the etched detector) shows a rough surface. Some track-like damages are also observable in the figure. In fact, these track-like damages are more easily observable under the optical microscope. Fig. 6 shows an optical-microscopic image of an etched LR 115 detector with a remaining active-layer thickness of $5.46 \mu \mathrm{m}$. The dimensions of the damages also match those recorded by the Form Talysurf shown in Fig. 4 It is also noted that track-like damages with depths up to $0.33 \mu \mathrm{m}$ are also detected under the atomic force microscope in LR 115 detectors etched for $30 \mathrm{~min}$ (Ho et al., 2003).

Therefore, these track-like damages are ubiquitous on the surface of etched LR 115 detectors, which can increase the 


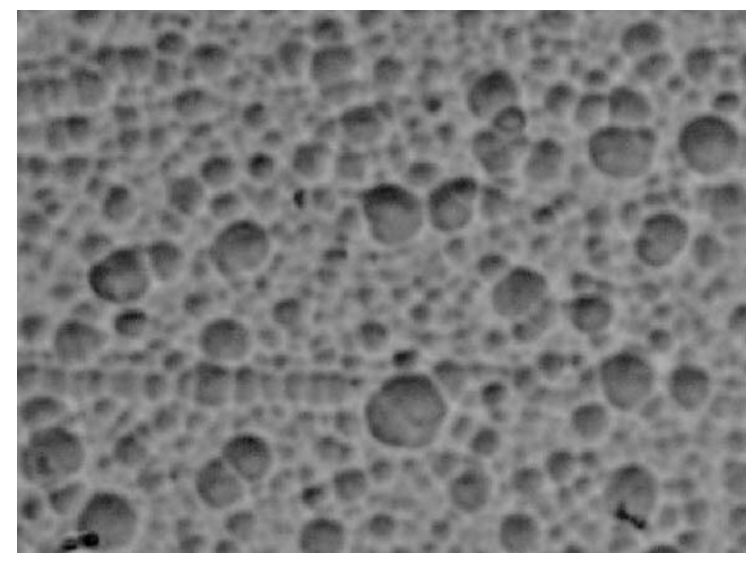

Fig. 6. An optical-microscopic image of an etched LR 115 detector with a remaining active layer thickness of $5.46 \mu \mathrm{m}$. The size of the image is $36.4 \times 27 \mu \mathrm{m}^{2}$.

inhomogeneity in the thickness of LR 115 detectors. When the thickness of the active layer drops to $\sim 5 \mu \mathrm{m}$, the standard deviation rises to $\sim 0.4 \mu \mathrm{m}$ and the coefficient of variation to $\sim 8 \%$. As this value is quite large, the thickness of the active layer in the LR 115 detector cannot be assumed to be homogeneous in general, and the associated uncertainties should be considered carefully when the detector is used for alpha spectroscopy.

\section{Acknowledgements}

The present research is supported by the CERG grants CityU1081/01P and CityU1206/02P from the Research Grant Council of Hong Kong.

\section{References}

Durrani, S.A., Bull, R.K., 1987. Solid State Nuclear Track Detection: Principles, Methods and Applications. Pergamon Press, Oxford.

Ho, J.P.Y., Yip, C.W.Y., Nikezic, D., Yu, K.N., 2003. Differentiation between tracks and damages in SSNTD under the atomic force microscope. Radiat. Meas., this issue doi: 10.1016/S1350-4487(03)00114-8.

Nikezić, D., Janicijević, A., 2002. Bulk etching rate of LR 115 detectors. Appl. Radiat. Isot. 57, 275-278. 